\title{
COMPARACIÓN DE MÉTODOS GRAVIMÉTRICOS Y TERMOGRAVIMÉTRICOS PARA LA OBTENCIÓN DEL PORCENTAJE DE RESIDUO DE ASFALTO EN UNA EMULSIÓN ASFÁLTICA
}

\section{COMPARISON OF GRAVIMETRIC AND THERMOGRAVIMETRIC METHODS FOR OBTAINING THE PERCENTAGE OF RESIDUE ASPHALT BITUMEN EMULSION}

Tec. Cindy Zúñiga Arava

Técnico Especializado Laboratorio de Ligantes Asfálticos

LanammeUCR, Costa Rica

E-mail: cindy.zuniga@ucr.ac.cr

\section{RESUMEN}

La caracterización de las emulsiones asfálticas incluye el ensayo de destilación para cuantificar el contenido de asfalto. Este ensayo se realiza desde hace mucho tiempo, tanto en Costa Rica como en otros países. Es un método tradicional y preciso, que ha representado una muy buena opción para la recuperación del asfalto residual de una emulsión asfáltica, pero consume mucho tiempo y requiere de la pericia del analista para identificar momentos clave de la separación.

En vista de que se pueden utilizar las nuevas tecnologías para la cuantificación de la composición de las emulsiones asfálticas, se propone aplicar técnicas de análisis termogravimétricos instrumentales.

La primera fase del proyecto consiste en determinar los parámetros que se deben utilizar en un analizador termogravimétrico (TGA, por sus siglas en inglés) para obtener resultados comparables con el método de destilación. Para lo cual se varían las rampas de temperatura y la velocidad de calentamiento en cada una de las muestras analizadas.

A pesar de que los resultados no presentan diferencias estadísticamente significativas, se percibe el efecto que tienen los cambios de parámetros en el análisis termogravimétricos en los resultados.

Los resultados obtenidos muestran que es posible utilizar los análisis termogravimétricos instrumentales para determinar el porcentaje de residuo asfáltico en la emulsión, pero además permite establecer las condiciones que deben definirse como parte de un procedimiento de análisis, según los resultados debe seleccionarse tasas de calentamiento de $10{ }^{\circ} \mathrm{C} / \mathrm{min} \mathrm{o}$ menores a $5^{\circ} \mathrm{C} / \mathrm{min}$. Asimismo se determina que las tasas de calentamiento moduladas no arrojan buenos resultados.

En la siguiente fase se compararán los métodos mencionados utilizando emulsiones fabricadas en ellaboratorio, considerando los parámetros definidos en esta fase.

PALABRAS CLAVE:

Termogravimetría, Termograma, Análisis térmico, Destilación de la emulsión asfáltica, Asfalto residual
Químico. Jorge Salazar Delgado

Unidad de Materiales y Pavimentos

LanammeUCR, Costa Rica

E-mail: jorge.salazardelgado@ucr.ac.cr

Ing. Ellen Rodríguez Castro

Jefe Laboratorio de Ligantes Asfálticos

LanammeUCR, Costa Rica

E-mail: ellen.rodriguez@ucr.ac.cr

\section{ABSTRACT}

The characterization includes asphalt emulsions assay to quantify the distillation bitumen content. This test is performed for a long time, both in Costa Rica and other countries. It is a traditional and accurate method, which has been a very good choice for the recovery of residual asphalt bitumen emulsion, but is time consuming and requires the skills of the analyst to identify key moments of separation.

Given that new technologies can be used to quantify the composition of asphalt emulsions, it intends to apply instrumental techniques thermogravimetric analysis The first phase of the project is to determine the parameters to be used in a thermogravimetric analyzer (TGA) to get comparable results with the method of distillation. For which the temperature ramp and the heating rate in each of the samples are varied.

Although the results show no statistically significant differences, the effect of parameter changes in the thermogravimetric analysis results in perceived.

The results show that it is possible to use the instrumental thermogravimetric analysis to determine the percentage of asphalt emulsion residue, but also allows establishing the conditions to be defined as part of a process of analysis should be selected according to the results heating rates $10^{\circ} \mathrm{C} / \mathrm{min}$ or less than $5^{\circ} \mathrm{C} / \mathrm{min}$. It also determined that rates of warming modulated not give good results. In the next phase of the project the methods will be compared using emulsions manufactured in the laboratory, considering the parameters defined in this phase.

\section{KEY WORDS:}

Thermogravimetry, Thermogram, Thermal Analysis, Asphalt emulsions distillation, Residual bitumen. 


\section{INTRODUCCIÓN}

La emulsión asfáltica se utiliza en diversos tratamientos de preservación y mantenimiento, tanto con agregado como sin agregado, desde hace más de 100 años (Mercado, y otros, 2008). Independientemente del tratamiento el objetivo es poder usar el material asfáltico a bajas temperaturas y que la capa de asfalto remanente cumpla con el propósito de la técnica que se está aplicando (Akzo Nobel, 2009).

Para determinar cuánto asfalto contiene la emulsión se utilizan los ensayos de evaporación o el ensayo de destilación (La Gaceta, 2005).

El ensayo de destilación de la emulsión asfáltica consiste en calentar una muestra de emulsión asfáltica gradualmente hasta $260^{\circ} \mathrm{C}$ por al menos una hora (ASTM, 2012). El calentamiento pretende separar el asfalto que contiene la emulsión del agua y del emulsificante. La cuantificación del contenido de asfalto en la emulsión se estima midiendo la masa de la emulsión antes y después del ensayo, con lo cual se determina el porcentaje de bitumen presente en la emulsión.

A pesar de que la destilación tarda una hora, la preparación y el enfriamiento posterior alarga el tiempo de ensayo a dos horas. Es por este motivo que se considera la posibilidad de aplicar técnicas de análisis termogravimétrico por medios instrumentales (TGA, por sus siglas en inglés) como una opción para sustituir el ensayo de destilación.

El método de destilación consiste en colocar 200 g de emulsión asfáltica en un contenedor diseñado para tal fin, donde se aplica calentamiento gradual hasta llegar en primera instancia a $215{ }^{\circ} \mathrm{C}$ en la parte superior. Luego se mueve la fuente de calor hacia la parte inferior del contenedor para alcanzar los $260{ }^{\circ} \mathrm{C}$. El objetivo es que el agua, el emulsificante y otros aceites, se evaporen y salgan del contenedor a través de un tubo de vidrio y un condensador de agua para ser separados del asfalto. El asfalto residual se mantiene en el contenedor y los gases condensados se almacenan en una probeta colocada a la salida del condensador. Una vez finalizada la destilación se debe acondicionar el contenedor a temperatura ambiente para ser pesado y por diferencia de masa calcular el porcentaje de asfalto presente en una emulsión asfáltica (ASTM, 2012). El calentamiento del ensayo requiere entre una hora y hora y media, a este tiempo se debe sumar el tiempo de preparación $y$ de enfriamiento del equipo.

Algunas de las ventajas que posee del método de destilación son:

- Datos muy confiables si el operador está entrenado adecuadamente en el método.

- El residuo de asfalto puede ser utilizado para otras pruebas.

- Esta prueba se aplica a diferentes tipos de emulsión asfáltica.

- El volumen de agua, emulsificante y otros materiales se puede cuantificar directamente.

Sin embargo también se pueden detectar desventajas en el método de destilación, dentro de las cuales se mencionan las siguientes:

- Es una prueba que consume mucho tiempo en el calentamiento y enfriamiento.

- La emulsión asfáltica es expuesta altas temperaturas de calentamiento y es posible que parte del asfalto sea arrastrado por el destilado.

- El resultado depende de la precisión del operador.

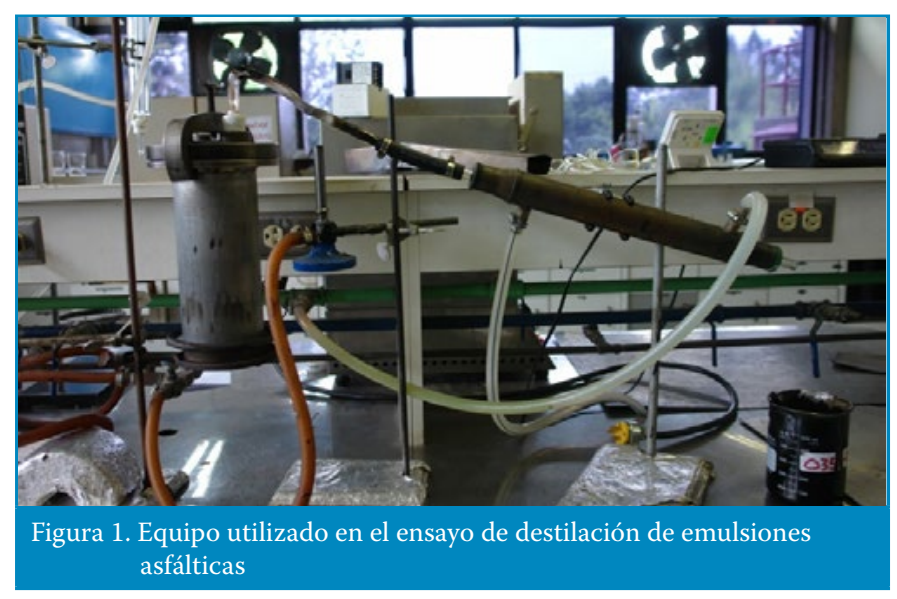

La termogravimetría es un estudio térmico desarrollado para identificar y medir los cambios físicos y químicos, que sufren los materiales cuando son expuestos a variaciones controladas de temperatura (Conesa Ferrer, 2000). Los análisis termogravimétricos han sido utilizados para el estudio de las reacciones primarias en la descomposición de materiales sólidos y líquidos mediante la aplicación de calor (Fraga Grueiro, 2001). Este es el caso de las emulsiones asfálticas, pues el propósito del ensayo de destilación es eliminar, principalmente, el agua y el emulsificante.

El análisis termogravimétrico registra la variación de la masa del material en estudio conforme se varía la temperatura a una tasa térmica constante (cambio de temperatura lineal). Como resultado del análisis termogravimétrico se obtienen los datos de cambio de masa con respecto a la temperatura o con respecto al tiempo y un termograma, el cual representa gráficamente las variaciones porcentuales de la masa (Rodríguez, y otros, 2012).

Este tipo de técnica es ampliamente utilizada en la caracterización cuantitativa y caracterización cinética de polímeros, carbón y arcillas, entre otros materiales. Incluso en Costa Rica, esta técnica es utilizada para el análisis de suelos, productos alimenticios y cultivos entre otras áreas, por lo que no se puede considerar como una técnica desconocida ni se puede negar la amplia gama de aplicaciones.

La aplicación de esta técnica no es inusual en los asfaltos, desde hace algún tiempo se ha utilizado para evaluar asfalto original y asfalto modificado (Leiva, y otros, 2014) tanto para 
verificar resistencias térmicas como una dispersión apropiada del material inorgánico al cuantificar su residuo. Sin embargo, esta técnica no ha sido explorada en la cuantificación de la composición de las emulsiones asfálticas, pero se ha utilizado para modelar la pérdida de agua en las emulsiones (Banerjee, y otros, 2012), por lo que se considera que el análisis termogravimétrico mediante equipo instrumental es una herramienta muy prometedora para conocer de forma rápida y precisa el porcentaje de residuo de una emulsión asfáltica.

Desde hace varios años se desarrolló el Analizador Termogravimétrico (TGA, por sus siglas en inglés). Este equipo permite cuantificar el cambio de masa de muestras muy pequeñas y un control más estricto de la tasa de calentamiento. Los cambios de masa y las temperaturas, quedan registrados en el software del equipo, así como en el termograma resultante.

Dentro de las ventajas de usar el TGA para estimar el residuo asfáltico se pueden mencionar:

- Muy poca cantidad de muestra, entre $10 \mathrm{mg}$ y $50 \mathrm{mg}$.

- Resultados muy precisos.

- En dos horas se tienen resultados analizados.

Pero también presenta algunas desventajas, dentro de las más importantes están:

- Es una prueba destructiva.

- No se puede analizar el asfalto residual.

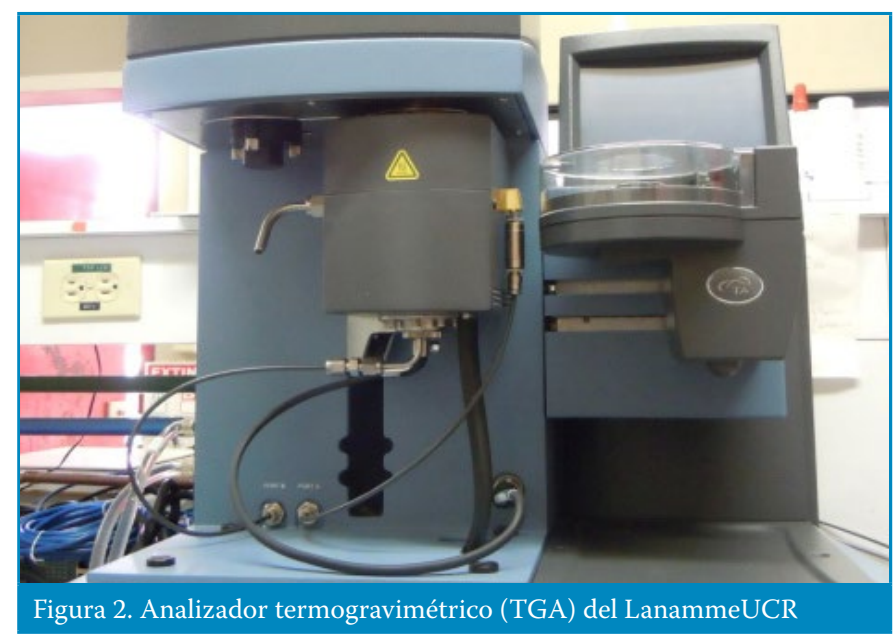

\section{OBJETIVOS}

El objetivo principal de esta fase del proyecto es determinar si es posible utilizar el análisis termogravimétrico para cuantificar el residuo de la emulsión asfáltica.

Como objetivos específicos se definen:

- Determinar el residuo asfáltico de las muestras emulsión usando el método de destilación.

- Determinar las condiciones de medición en el TGA de manera que se pueda establecer una rampa confiable de temperatura.
- Establecer si existe correlación entre el residuo asfáltico que se obtiene con el método de destilación y los cambios de masa que se detectan en el termograma.

\section{METODOLOGÍA}

Los resultados que se analizaron en este estudio corresponden solo a los residuos de los destilados. Las destilaciones que se efectuaron para cuantificar el porcentaje de residuo asfáltico, se realizaron por duplicado a cada muestra de acuerdo con el método establecido en la norma ASTM D 6997.

De igual manera se realizaron por lo menos dos réplicas en el TGA de cada una de las muestras, tanto para definir las condiciones de la medición como para comprobar la repetibilidad del método.

El análisis se realizó en el TGA del LanammeUCR marca TA Instruments serie Q5000, que cuenta con una balanza con una precisión de $\pm 1 \mu \mathrm{g}$ y con un horno infrarrojo capaz de hacer mediciones desde los $5{ }^{\circ} \mathrm{C}$ hasta los $1000{ }^{\circ} \mathrm{C}$, con una velocidad de calentamiento de $0{ }^{\circ} \mathrm{C} / \mathrm{min}$ (cuando se generan isotermas) hasta $300^{\circ} \mathrm{C} / \mathrm{min}$ y con una precisión de $\pm 1 \%$. Las mediciones se pueden realizar en atmósferas inertes como el argón y el nitrógeno o en presencia de oxígeno, con una razón de flujo entre $10 \mathrm{~mL} / \mathrm{min}$ y $100 \mathrm{~mL} / \mathrm{min}$ con una precisión de $\pm 5 \mathrm{~mL} / \mathrm{min}$.

Como no existía un método previamente establecido para el análisis termogravimétrico de la emulsión se utilizaron distintas rampas de calentamiento y diferentes temperaturas finales con el propósito de optimizar el método.

\subsection{Muestras Utilizadas}

Para este estudio se seleccionaron seis muestras de emulsión asfáltica de rompimiento rápido, pues son las que se distribuyen en Costa Rica, de acuerdo con sus características se clasifica como CRS-1 (La Gaceta, 2005). Este tipo de emulsiones tienden a romper muy rápido, especialmente si no se almacenan adecuadamente. Por lo que antes de iniciar cualquier prueba a una emulsión asfáltica tipo CRS-1 se debe agitar suavemente para homogenizar la muestra y evitar la formación de burbujas.

\subsection{Procedimiento de ensayo}

Como se mencionó anteriormente, el ensayo de destilación se realizó de acuerdo con el procedimiento establecido y acreditado en el LanammeUCR conforme con la norma ASTM D 6997, el cual sigue el procedimiento descrito en la introducción.

Actualmente, no existe una norma que indique los parámetros que se deben definir en el TGA para analizar emulsiones asfálticas, por lo que los parámetros de medición 
se seleccionan basándose en la experiencia de uso del equipo y el conocimiento adquirido durante varios años de análisis de la emulsión asfáltica que se utiliza en Costa Rica así como de la emulsión producida en laboratorio.

Se deben establecer parámetros que generen un método que proporcione resultados comparables con el método de destilación y que además sea repetible y reproducible.

Bajo estas premisas, se seleccionó un tamaño de muestra usual entre $10 \mathrm{mg}$ y $50 \mathrm{mg}$ de emulsión asfáltica, que se colocó en el contenedor de muestra de platino, como se observa en la Figura 3. Y se utilizan diferentes rampas de medición tomando en cuenta parámetros de producción y de manipulación de las emulsiones asfálticas.

Durante las mediciones se hicieron ajustes con el propósito de valorar distintos parámetros y detectar aquellos que influyen en los resultados.

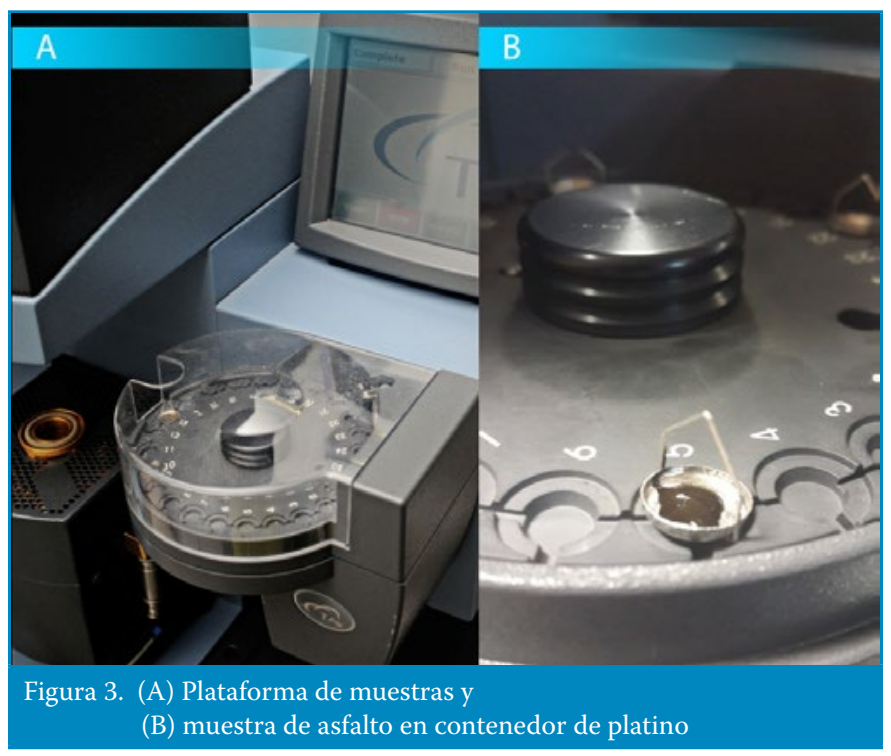

Los resultados de destilación reportados corresponden al promedio de dos réplicas y los resultados de TGA para cada muestra es el resultado del promedio de dos réplicas por lo menos de las mediciones realizadas en un mismo día.

En la Tabla 1, se resumen las condiciones de ensayo utilizadas en cada una de las muestras analizadas.

\begin{tabular}{|c|c|c|c|}
\hline \multirow{2}{*}{$\begin{array}{c}\text { Identificación } \\
\text { de la } \\
\text { muestra }\end{array}$} & $\begin{array}{c}\text { Tasa de } \\
\text { calentamiento }\end{array}$ & $\begin{array}{c}\text { Temperatura } \\
\text { final de la rampa }\end{array}$ & \multirow{2}{*}{ Atmósfera } \\
\hline & $\left({ }^{\circ} \mathrm{C} / \mathrm{min}\right)$ & $\left({ }^{\circ} \mathrm{C}\right)$ & \\
\hline 1 & 2 & 400 & \multirow{10}{*}{ 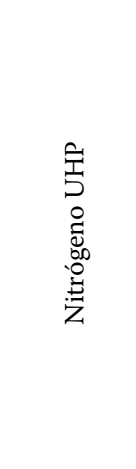 } \\
\hline 2 & 5 & 600 & \\
\hline 3 & 7 & 550 & \\
\hline 4 & 10 & 990 & \\
\hline 5 & 10 & 980 & \\
\hline \multirow{5}{*}{6} & 10 & 100 & \\
\hline & 3 & 170 & \\
\hline & 1 & 215 & \\
\hline & 5 & 260 & \\
\hline & 15 & 800 & \\
\hline
\end{tabular}

Tabla 1. Condiciones de análisis en el TGA

\section{RESULTADOS}

En las siguientes figuras se muestran algunos de los termogramas obtenidos. El TGA utilizado permite estimar la curva de la segunda derivada (líneas en color azul) del cambio de masa en función de la temperatura, esto facilita la visualización e identificación de los cambios más significativos mediante picos.

Las mediciones que se efectuaron durante varios días demuestran la pérdida natural de agua.

(véase figuras 4, 5, 6 y 7 en pagina 8 y 9)

En la Tabla 2 se presentan los resultados del residuo asfáltico obtenido para cada muestra y mediante los dos métodos utilizados.

\begin{tabular}{|c|c|c|}
\hline \multirow{2}{*}{ Muestra } & \multicolumn{2}{|c|}{ Residuo (\%) } \\
\cline { 2 - 3 } & Destilación & TGA \\
\hline 1 & 60,60 & 58,96 \\
\hline 2 & 61,70 & 65,73 \\
\hline 3 & 62,00 & 63,96 \\
\hline 4 & 61,70 & 63,75 \\
\hline 5 & 61,90 & 64,24 \\
\hline 6 & 62,80 & 60,23 \\
\hline
\end{tabular}

Tabla 2. Porcentajes de residuo asfáltico mediante los métodos de destilación y TGA 


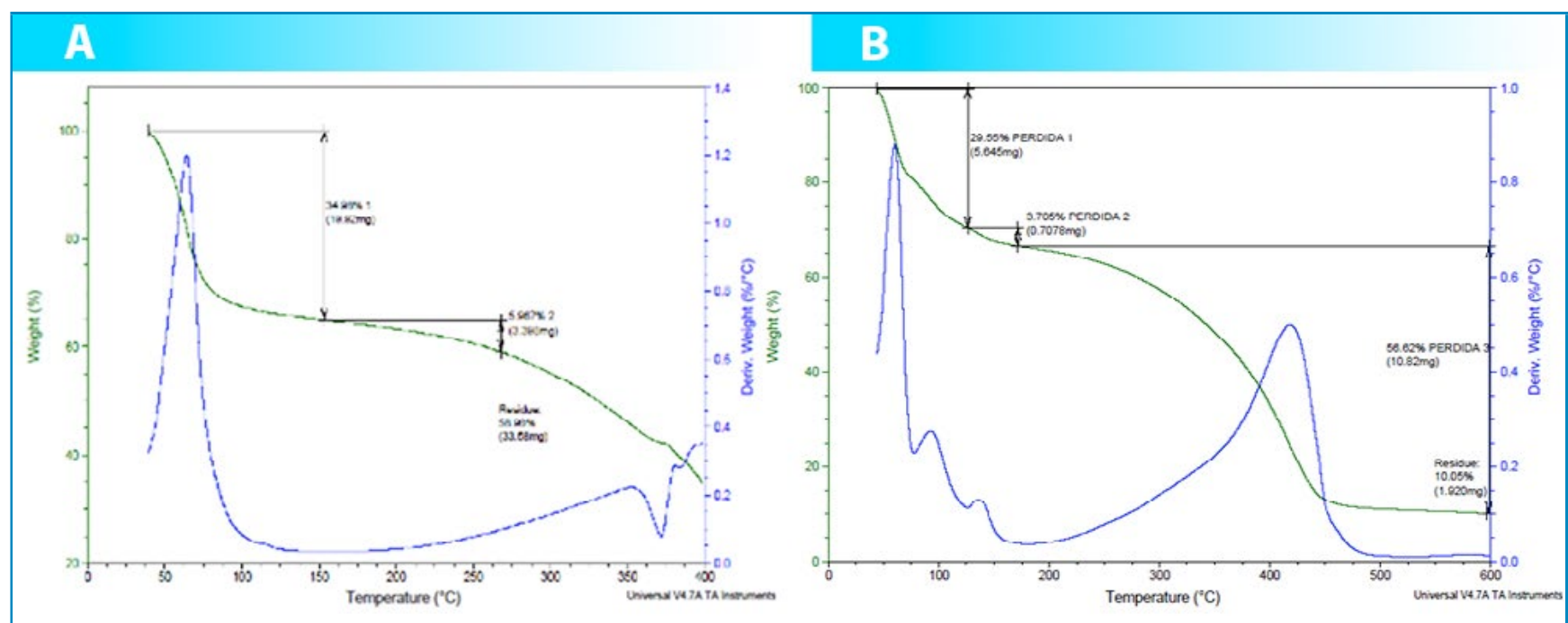

Figura 4. Termogramas de una de las réplicas de la (a) Muestra 1 rampa de temperatura $40{ }^{\circ} \mathrm{C}$ a $400{ }^{\circ} \mathrm{C}$ en atmósfera inerte a $2{ }^{\circ} \mathrm{C} / \mathrm{min}$ y (b) Muestra 1 rampa de temperatura $40{ }^{\circ} \mathrm{C}$ a $600{ }^{\circ} \mathrm{C}$ en atmósfera inerte a $5{ }^{\circ} \mathrm{C} / \mathrm{min}$
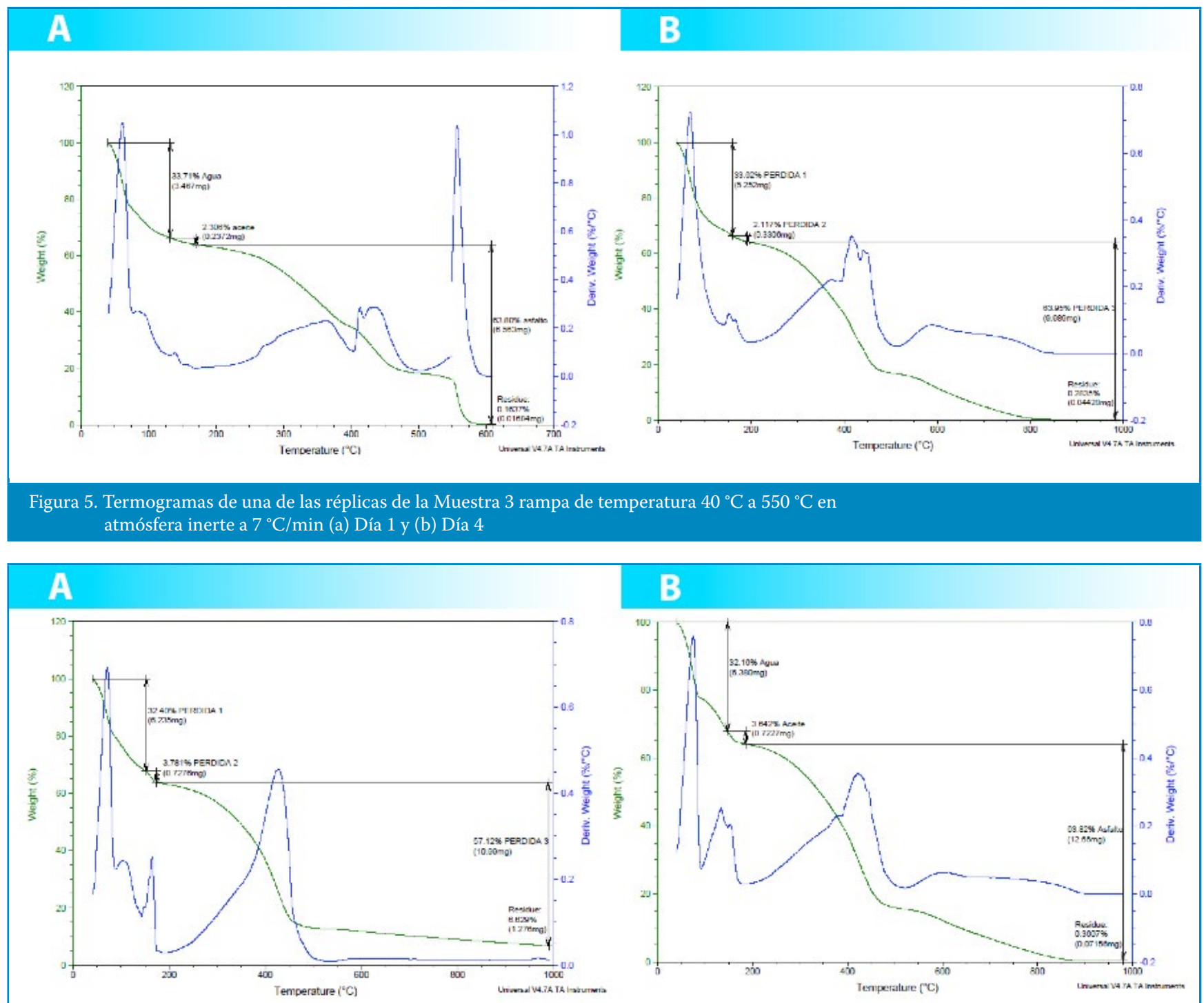

Figura 6. Termogramas de una de las réplicas de (a) Muestra 4 rampa de temperatura $40{ }^{\circ} \mathrm{C}$ a $9900^{\circ} \mathrm{C}$ en atmósfera inerte a $10{ }^{\circ} \mathrm{C} / \mathrm{min}$ Día 1 y (b) Muestra 5 rampa de temperatura $40^{\circ} \mathrm{C}$ a $990^{\circ} \mathrm{C}$ en atmósfera inerte a $10^{\circ} \mathrm{C} /$ min Día 1 


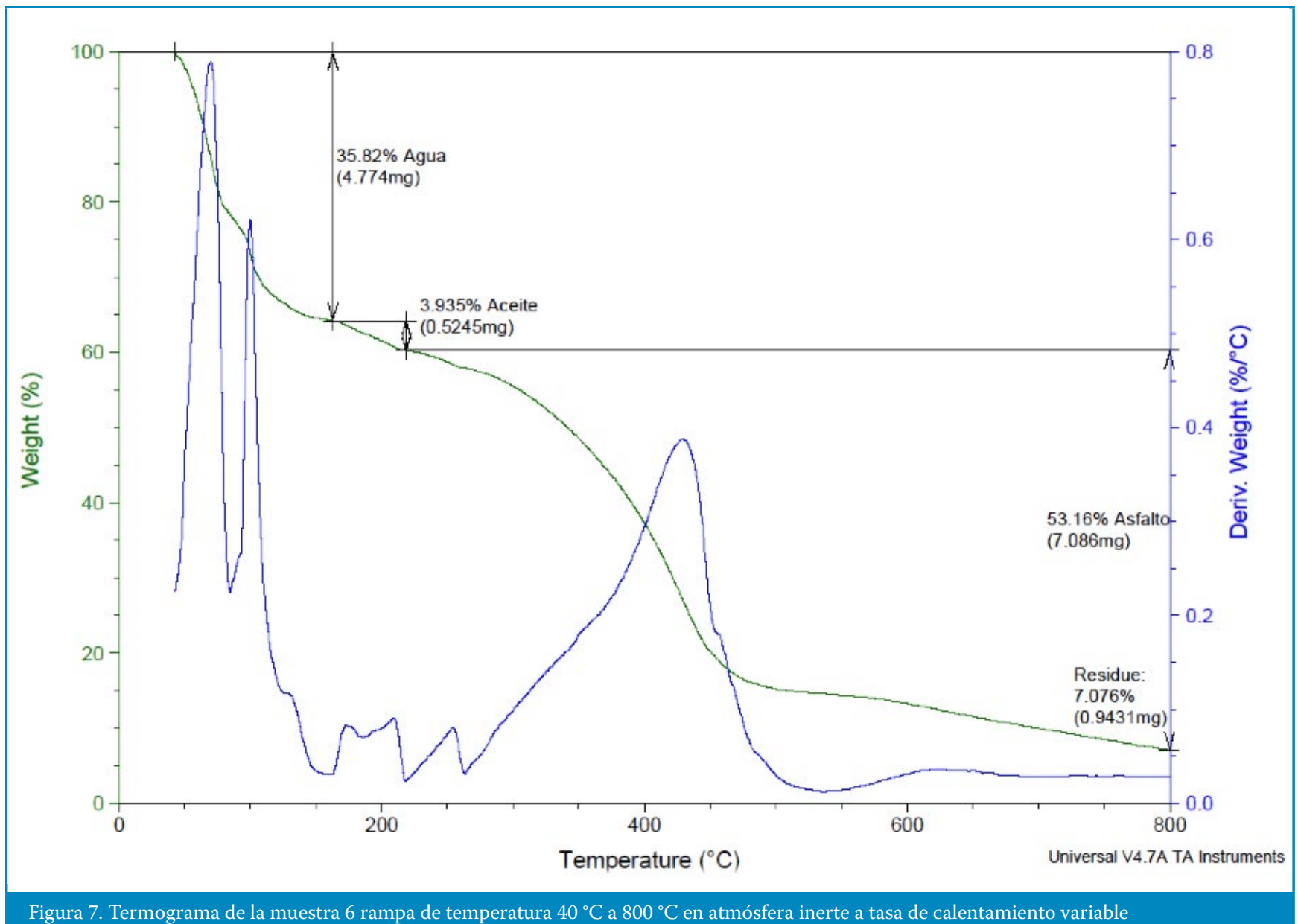

\section{DISCUSIÓN}

Durante el estudio se valoraron los parámetros de ensayo en el TGA en cada una de las muestras como se resume en la Tabla 1. A continuación se detalla el análisis realizado para definir los parámetros de acuerdo con los resultados que se obtenían.

En la medición de la muestra 1 se seleccionó una temperatura inicial de la rampa de $40^{\circ} \mathrm{C}$, considerando que la emulsión se debe acondicionar a $50^{\circ} \mathrm{C}$ en el ensayo de destilación. Como temperatura final se selecciona $400{ }^{\circ} \mathrm{C}$ con el propósito de observar el comportamiento de la emulsión a temperaturas superiores a la temperatura de $260{ }^{\circ} \mathrm{C}$ que corresponde a la máxima temperatura alcanzada en el ensayo de destilación. Adicionalmente se establece una rampa de $2{ }^{\circ} \mathrm{C} / \mathrm{min}$. Para todas las mediciones se elige una atmósfera inerte de nitrógeno UHP.

Con la intención de optimizar los resultados, para la muestra 2 se aumenta la temperatura final de la rampa hasta $600{ }^{\circ} \mathrm{C}$ y se aumenta la velocidad de calentamiento a $5{ }^{\circ} \mathrm{C} / \mathrm{min}$. Es importante mencionar que a los $600{ }^{\circ} \mathrm{C}$ se espera que se hayan quemado toda la materia orgánica de la muestra.
Debido a que a $600{ }^{\circ} \mathrm{C}$ no se detecta cambio de masa para las mediciones de la muestra 3 se reduce la rampa a una temperatura final a $550{ }^{\circ} \mathrm{C}$ y se aumenta la tasa de calentamiento. Adicionalmente se hacen mediciones durante cinco días consecutivos para determinar si los resultados son reproducibles en el tiempo.

Para las muestras 4 y 5 se opta por la calcinación, por lo que se aumenta la temperatura hasta $990{ }^{\circ} \mathrm{C}$ y $980{ }^{\circ} \mathrm{C}$, respectivamente y se aumenta la velocidad de calentamiento a $10^{\circ} \mathrm{C} / \mathrm{min}$, manteniendo los demás parámetros constantes.

Finalmente, después de analizar todos los parámetros, para la muestra 6 se decide elaborar una rampa de temperatura más compleja que permita observar y cuantificar con mayor detalle cada uno de los cambios que suceden durante el calentamiento de la muestra.

En la primera sección de la rampa se aumenta la temperatura desde $40{ }^{\circ} \mathrm{C}$ hasta $100{ }^{\circ} \mathrm{C}$ a una tasa de calentamiento de $10^{\circ} \mathrm{C} / \mathrm{min}$. La segunda sección finaliza en $170^{\circ} \mathrm{C}$, el propósito es evaporar completamente el agua de la muestra, en este caso de usa una tasa de $3{ }^{\circ} \mathrm{C} / \mathrm{min}$ para asegurarse de que se elimine completamente el agua. Posteriormente se aumenta a $215^{\circ} \mathrm{C}$ lentamente $\left(1^{\circ} \mathrm{C} / \mathrm{min}\right)$ para determinar la pérdida de 
aceites, y desde este punto se aumenta a $5{ }^{\circ} \mathrm{C} / \mathrm{min}$ hasta 260 ${ }^{\circ} \mathrm{C}$ para completar la destilación y determinar el contenido de los materiales volátiles más pesados. En el rango de $215{ }^{\circ} \mathrm{C}$ hasta $260{ }^{\circ} \mathrm{C}$, se determina el residuo de asfalto junto con el material que se pierde hasta los $800^{\circ} \mathrm{C}$, temperatura a la cual se llega a una tasa de $15{ }^{\circ} \mathrm{C} / \mathrm{min}$.

Los resultados obtenidos en este estudio nos permiten comparar los dos procedimientos. Se analizan las réplicas y se estiman los promedios. A pesar de que no es una misma muestra, la producción de emulsión utiliza la misma dosis y materiales, por lo que realiza un análisis de varianza, suponiendo que la muestra proviene de una sola fuente.

La comparación de varianzas reporta un valor de $\mathrm{F}$ de 0,862 , mucho menor al tabulado $\left(\mathrm{F}_{(1,10,11) \alpha 0,05}=4,96\right)$, lo cual indica que a pesar de que el rango de resultados obtenidos con el TGA es mayor a los obtenidos con el método de destilación, las diferencias no son significativas.

La Figura 8 permite ver de manera gráfica la variabilidad y asimetría de estos porcentajes de residuo.

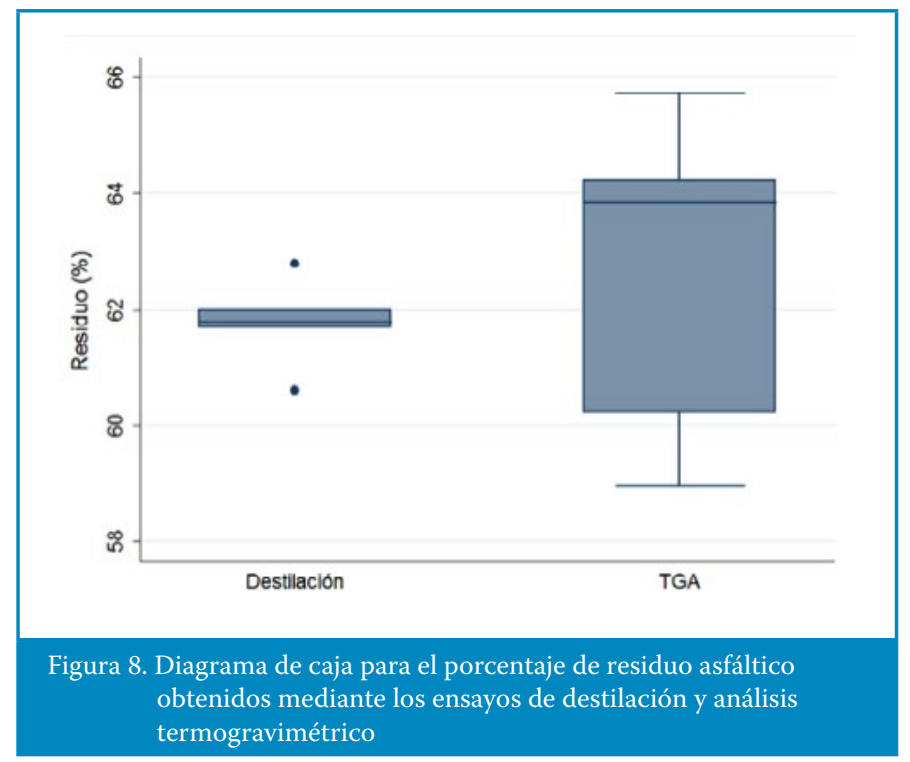

Es claro que el método de destilación presenta menor variabilidad en sus resultados que el método que utiliza el TGA.

Los resultados obtenidos para cada muestra se comparan individualmente en la Figura 9.

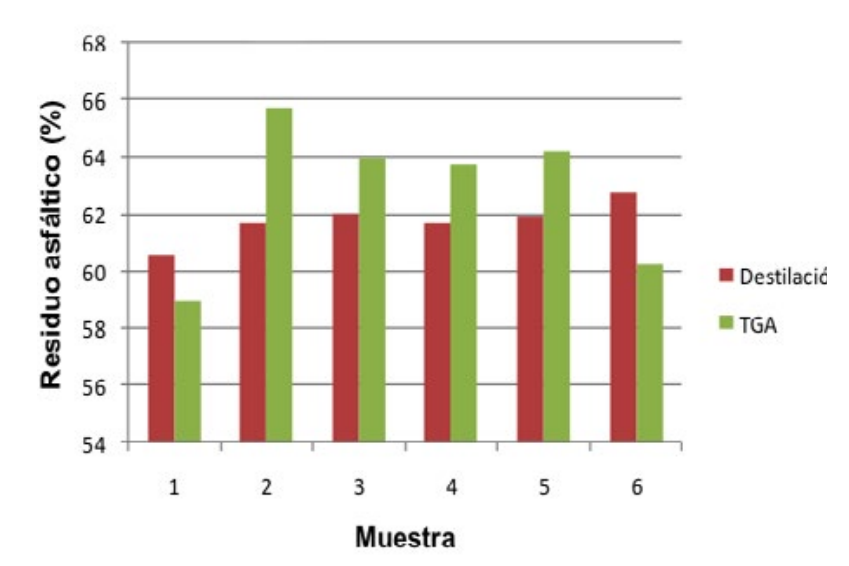

Figura 9. Comparación entre los resultados de residuo asfáltico obtenidos para cada muestra aplicando cada método

Los resultados obtenidos con el método termogravimétrico presentaron un valor menor al obtenido con destilación para las muestras 1 y 6 , en los otros casos, el resultado es mayor. En la Figura 10 se muestran las diferencias obtenidas entre los métodos para cada muestra.

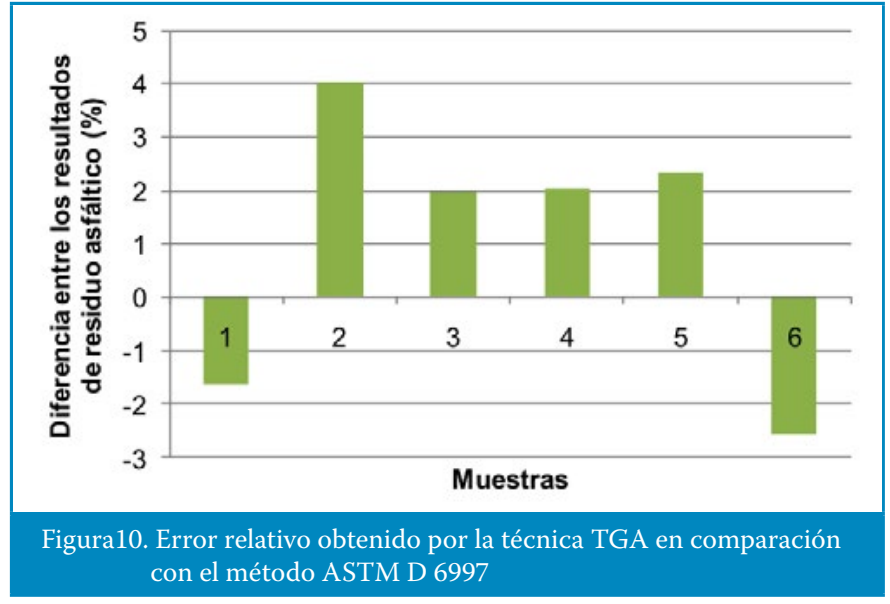

Se observa que los resultados que tienen una diferencia menor corresponden a las muestras 1,3 y 4 . Sin embargo en la muestra 1 el resultado de destilación es menor al resultado de TGA.

El principal cambio en estas muestras es la tasa de calentamiento. En la muestra 1 se utilizó una tasa baja de $2{ }^{\circ} \mathrm{C} /$ min y en las muestras 3 y 4 tasas de $7{ }^{\circ} \mathrm{C} /$ min y $10{ }^{\circ} \mathrm{C} / \mathrm{min}$, respectivamente. El peor resultado se obtiene cuando se aplica una tasa de calentamiento de $5{ }^{\circ} \mathrm{C} / \mathrm{min}$. Parece que la rampa de calentamiento no genera tampoco resultados cercanos a los que se obtienen con la destilación.

No obstante, no puede afirmarse de que el valor obtenido por la destilación es exacto, por lo que no puede descartarse aún que el resultado de residuo asfáltico estimado con el TGA sea el verdadero. 
Con respecto a los análisis realizados a las emulsiones durante varios días consecutivo, se observa que el porcentaje de pérdida que se produce cerca de los $100{ }^{\circ} \mathrm{C}$ se reduce, lo cual es un comportamiento esperado, pues el agua que contiene la emulsión se evapora, esto significa que la pérdida de masa detectada por el equipo es menor con respecto a días anteriores cuando el contenido de agua es mayor.

Se deben realizar más pruebas al respecto para determinar la velocidad con la que la emulsión pierde el agua y establecer que otras variables ambientales influyen en este proceso.

\section{CONCLUSIONES}

- La destilación es un método de ensayo confiable, reconocido y que utiliza equipo de laboratorio sencillo, pero requiere tiempos de acondicionamiento antes y después, del ensayo, prolongados. Además depende significativamente de la pericia del técnico.

- La técnica termogravimétrica es una opción viable para sustituir el ensayo de destilación de la emulsión asfáltica reduciendo el tamaño de muestra y el tiempo de respuesta en que se obtienen los resultados.

- La termogravimetría requiere de equipo más caro y sensible, que utiliza insumos como nitrógeno, que encarece el ensayo.

- La termograviometría permite detectar los cambios que se producen a cada temperatura, lo cual no es posible con el método de destilación, permite además definir los parámetros de ensayo con mayor precisión y reduce la intervención del analista.

- Con respecto a los resultados de este estudio:

- Parece que la rampa de calentamiento influye significativamente en los resultados obtenidos, los valores más cercanos a la destilación se reportan con rampas de calentamiento bajas y altas, tasas $5{ }^{\circ} \mathrm{C} / \mathrm{min}$ se alejan de los resultados de la destilación.

- Las rampas de calentamiento moduladas no producen los resultados esperados, es posible que utilizar tasas de $5{ }^{\circ} \mathrm{C} / \mathrm{min}$ en uno de los módulos tenga un efecto negativo en los resultados.

- Se deben seguir ajustando los parámetros del método de TGA, para lo cual es recomendable usar emulsiones fabricadas en el laboratorio, para de esta manera conocer el contenido de asfalto real.

- Sería valioso realizar un estudio utilizando la termogravimetría en la que se analice con más detalle la pérdida de agua de la emulsión con respecto al tiempo y correlacionarlo con las condiciones ambientales.

\section{BIBLIOGRAFÍA}

Akzo Nobel (2009). Productos y Aplicaciones. Chicago, EEUU.

American Standard for Testing and Material (2012). ASTM D 6997 Standard Test Method for Distillation of Emulsified Asphalt. Penslvania, EEUU: Autor.

American Standard for Testing and Materials. ASTM E1131-08 Standard Method for Compositional Analysis by Thermogravimetry. Penslvania, EEUU: Autor.

Banerjee, A., de Fortier Smit A. y Prozzi, J. (2012). Modeling the effect of environmental factors on evaporative water loss in asphalt emulsions for chip seal applications Revista Construction and Building Materials. 27(1), 158-164.

Conesa-Ferrer, J.A. (2000). Curso básico de análisis térmico. Alicante, España: Club Universitario [Book]. - San Vicente : Club Universitario.

Fraga-Grueiro, L. (2001). Estudio cinético, dinamomecánico y termogravimétrico del sistema epoxídico BADGE $(\mathrm{n}=0) / \mathrm{m}$-XDA, mediante las técnicas de análisis térmico : DSC, DMA y TGA. Construcción de un diagrama TTT. (Tesis inédita de doctorado). Universidad de Santiago de Compostela, España.

La Gaceta (20 de diciembre del 2005). Reglamento Técnico Centroamericano RTCA 75.01.22:04 Productos de Petróleo. Asfaltos. Especificaciones. 245. San José, Costa Rica.

Leiva-Villacorta, F., Aguiar-Moya, J.P., Villegas-Villegas, R.E., SalazarDelgado, J. y Loría-Salazar, G. (2014) Nano-materiales en el desempeño del Asfalto. Jornadas del asfalto. - San Pedro, Costa Rica.

Mercado, R., Bracho, C. y Avendaño, J. (2008). Emulsiones asfálticas: Usos-Rompimiento. Cuadernos FIRP S365-A. Mérida, Venezuela: Universidad de los Andes.

Rodríguez-Castro, E. y Villegas-Villegas, R.E. (2012). Caracterización de polímeros aplicando el método. Revista de Métodos y Materiales. 1 (12), 25-32. 